\title{
S100A4 is upregulated via the binding of c-Myb in methylation-free laryngeal cancer cells
}

\author{
JIA LIU ${ }^{1,2}$, ZHEN-MING XU ${ }^{3}$, GUANG-BIN QIU ${ }^{4}$, ZHI-HONG ZHENG ${ }^{2}$, KAI-LAI SUN ${ }^{1}$ and WEI-NENG FU ${ }^{1}$ \\ ${ }^{1}$ Department of Medical Genetics, China Medical University, Shenyang 110001; ${ }^{2}$ Laboratory Animal Center, \\ China Medical University, Shenyang 110001; ${ }^{3}$ Department of Otolaryngology, The 463 Hospital of PLA, \\ Shenyang 110007; ${ }^{4}$ Department of Laboratory Medicine, No. 202 Hospital of PLA, Shenyang 110003, P.R. China
}

Received September 12, 2013; Accepted October 11, 2013

DOI: $10.3892 /$ or.2013.2824

\begin{abstract}
DNA hypomethylation is correlated with the overexpression of the S100A4 gene in several types of cancers including laryngeal cancer, but the molecular mechanism is unknown. We speculated that the methylation status of the promoter affects its binding to the corresponding transcription factors. In the present study, luciferase reporter assay results indicated that the sequences $-485-+73$ and $-486--530$ of the $5100 A 4$ promoter may harbor the positive and negative cis-acting elements, respectively; and moreover, the luciferase activity promoted by the sequence $-485-+73$ increased and the S100A4 gene was significantly upregulated in 5-Aza-induced HEp2 cells. This implies that the methylation status of the sequence is important in regulating the expression of S100A4. Four transcription factor binding motifs including c-Myb, $\mathrm{C} / \mathrm{EBp} \alpha, \mathrm{Ap} 2$ and Msx-1 in the region were predicted by P-Match software. c-Myb and C/EBp $\alpha$ but not Ap2 and Msx-1 were confirmed by EMSA and ChIP as transcription factors of S100A4. The decreased luciferase activity in methylation-free HEp2 cells transfected by the mutant c-Myb motif related to the methylated cytosine suggests that the hypomethylation of the c-Myb motif upregulates the S100A4 expression in laryngeal cancer.
\end{abstract}

\section{Introduction}

Head and neck squamous cell carcinoma (HNSCC) is the eighth leading cause of cancer-related mortality worldwide, and laryngeal squamous cell carcinoma (LSCC) is a major type of HNSCC (1). In China, the incidence of LSCC has been gradually increasing, particularly in the Northeast. LSCC is a heterogeneous disease involving deregulation of multiple pathways involved in cellular differentiation, the cell cycle,

Correspondence to: Professor Wei-Neng Fu, Department of Medical Genetics, China Medical University, 92 Beier Road, Heping, Shenyang 110001, P.R. China

E-mail:wnfu@mail.cmu.edu.cn

Key words: laryngeal squamous cell carcinoma, S100A4, c-Myb, DNA methylation apoptosis, angiogenesis and metastasis (2). Despite advancement in local control and overall improvement in the quality of life achieved with the use of combined modality therapies, the survival rates for LSCC patients have not improved significantly over the past 2 decades. Therefore, the current research is often focused on the identification of useful biologic and molecular markers for the diagnosis and therapy of $\operatorname{LSCC}(3,4)$.

As one of the epigenetic types, DNA methylation can cause the silencing of gene transcription, genetic imprinting, genomic stability and $\mathrm{X}$ chromosome inactivation (5-7). Aberrant methylation of genes controlling the cell cycle, proliferation, apoptosis, metastasis, drug resistance and intracellular signaling has been identified in multiple types of cancers (8-10). Tumor-suppressor genes are often inactivated in cancer tissues by hypermethylation within $\mathrm{CpG}$ islands $(11,12)$. Hypomethylation usually leads to the activation of oncogenes in cancer tissues $(13,14)$. In our previous study, we found that the $5100 A 4$ gene is overexpressed in laryngeal cancer tissues and that RNAi S100A4 decreased the invasiveness of HEp2 cells, which implies that $S 100 A 4$ is involved in the progression of LSCC. We also found significant hypomethylation within the promoter region of S100A4 in LSCC tissues when compared to that in paired adjacent tissues. Statistical analysis showed the association of DNA hypomethylation and the overexpression of the S100A4 gene in laryngeal cancer tissues (15). However, the exact mechanism remains unclear. We speculated that the methylated sites of the S100A4 promoter affects the binding of the corresponding transcription factors to S100A4. In the present study, we identified the putative transcription factors that bind to S100A4 and explored the effect of hypomethylation on the binding.

\section{Materials and methods}

Cell culture and reagents. The human laryngeal carcinoma cell line HEp2 (Cell Biology Institute of Shanghai, Chinese Academy of Science) was maintained in RPMI-1640 (Gibco-BRL, Los Angeles, CA, USA) with 10\% fetal bovine serum, 100 units/ $\mathrm{ml}$ penicillin and $100 \mu \mathrm{g} / \mathrm{ml}$ streptomycin in a humidified atmosphere at $37^{\circ} \mathrm{C}$ with $5 \% \mathrm{CO}_{2} .5^{\prime}$-Aza-2'-deoxycytidine (5-Aza) was purchased from Sigma-Aldrich (St. Louis, MO, USA). Cells were treated with $4 \mu \mathrm{M} 5$-Aza for $72 \mathrm{~h}$. PGL3basic, pRL-TK and Dual-Luciferase reporter assay system 
Table I. Primer sequences used in the generation of the related constructs.

\begin{tabular}{ll}
\hline Primer name & \multicolumn{1}{c}{ Sequence: ' $^{\prime}$ to $3^{\prime}$} \\
\hline P0 & TTTAAGCTTTGCTCTGGGCAGTGAACAT \\
P1 & TTTGGTACCGCTGGGACTACAGGCTAC \\
P2 & TTTGGTACCGTGCCCATCTCATCCAG \\
P3 & TTTGGTACCGTGCCCACCTGGGAACA \\
P4 & TTTGGTACCTCAGCCCACAGCAGGAAG \\
P5 & TTTGGTACCCACACACACATGCACGTAAG \\
P6 & TTTGGTACCTGAGCAAGTGACTGAA \\
P7 & TGGGCTTGCACATTCTGTTGCTATAGTACG \\
P8 & TGAGATGTGGGCTTGCACATGTTGCTATAGTACGTGTTGGT \\
P9 & ACCAACACGTACTATAGCAACATGTGCAAGCCCACATCTCA \\
P10 & TTTGGTACCGGCTCATGTTTGCTGGGTT \\
P11 & GGCTCATGTTTGCTGGGTTGTACACTAAGGAGCAGGAAGC \\
P12 & ACTGGCTCATGTTTGCTGGGGAGCAGGAAGCAAAGGAAAGGCA \\
P13 & TGCCTTTCCTTTGCTTCCTGCTCCCCAGCAAACATGAGCCAGT
\end{tabular}

were obtained from Promega Corporation (Madison, WI, USA). The Plasmid Miniprep kit and Lipofectamine ${ }^{\mathrm{TM}} 2000$ transfection reagents were purchased from Invitrogen Life Technologies (Carlsbad, CA, USA). The primary antibodies, protein $\mathrm{A} / \mathrm{G}$-agarose and the anti-IgG antibody used in the present study were purchased from Santa Cruz Biotechnology, Inc. (Santa Cruz, CA, USA).

Plasmid construction. Human genomic DNA was isolated from HEp2 cells using a genomic DNA isolation kit (Beyotime, Jiangsu, China) according to the manufacturer's instructions. Over $1.2 \mathrm{~kb}$ fragment of the S100A4 5'-flanking sequence was amplified using Probest Taq DNA polymerase (Takara, Japan). KpnI and XhoI restriction sites were integrated into the fragment above using primers P0 and P1 (Table I). The fragment was then cloned into the plasmid PGL3-Basic, named P1283 (-1283/+73). The deletion constructs P970 (-970/+73), P912 (-912/+73), P842 (-842/+73), P530 (-530/+73) and P485 $(-485 /+73)$ were created by PCR using P1283 as a template and the oligonucleotides P2 to P6 as primers. The reporter constructs including point mutations and deletions at potential c-Myb and $\mathrm{C} / \mathrm{EBP} \alpha$ binding sites were generated using PCR-based Megaprimer technique as previously described (16). Primer P7 was used for the construction of the mutant c-Myb vector (pcMyb-M), P8 and P9 for the deletion cMyb vector (pcMyb-D), P10 for the C/EBP $\alpha$ wild-type vector, $\mathrm{P} 11$ for the mutant $\mathrm{C} / \mathrm{EBP} \alpha$ vector $(\mathrm{pC} / \mathrm{EBP} \alpha-\mathrm{M}), \mathrm{P} 12$ and $\mathrm{P} 13$ for the deletion $\mathrm{C} / \mathrm{EBP} \alpha$ vector $(\mathrm{pC} / \mathrm{EBP} \alpha-\mathrm{D})$. All constructs were sequenced in a capillary automatic sequencer (ABI PRISM ${ }^{\circledR} 3100$ Genetic Analyzer; Applied Biosystems). All primer sequences are listed in Table I.

Transient transfection and luciferase assays. HEp2 cells were seeded in 24-well plates, grown to $60-80 \%$ confluence, and transfected by the constructs using Lipofectamine ${ }^{\mathrm{TM}} 2000$ according to the manufacturer's instructions (Invitrogen Life Technologies). pRL-TK plasmid containing the Renilla lucif- erase gene was used as the internal control in each experiment. After $24 \mathrm{~h}$ of transfection, the cells were incubated with $4 \mu \mathrm{M}$ 5-Aza for an additional 3 days. Firefly and Renilla luciferase activities were determined using a Lumat LB 9507 luminometer (Bethold Technologies, Bad Wildbad, Germany).

Semi-quantitative $R T$-PCR. Total RNA was isolated by TRIzol reagent according to the manufacturer's instructions, and cDNA was reversibly transcribed from the isolated RNA using an AMV RNA PCR kit (Takara, Japan) in line with the standard operation protocol. The primer sequences were as follows: S100A4 forward, 5'-CCCTGGATGTGATGGTGTC-3' and reverse, 5'-CTCGTTGTCCCTGTTGCTG-3', which were expected to produce a 185 -bp DNA fragment; $\beta$-actin forward, 5'-CCAGATCATGTTTGAGACCT-3' and reverse, 5'-TTGAAGGTAGTTTCGTGGAT-3', which were expected to produce a 480-bp DNA fragment. The PCR reaction was performed in a $25-\mu 1$ reaction system, starting with denaturation at $94^{\circ} \mathrm{C}$ for $4 \mathrm{~min}$, then 30 cycles of denaturation at $94^{\circ} \mathrm{C}$ for $30 \mathrm{sec}$, annealing at $55^{\circ} \mathrm{C}$ for $30 \mathrm{sec}$, extension at $72^{\circ} \mathrm{C}$ for $45 \mathrm{sec}$, followed by an extra extension at $72^{\circ} \mathrm{C}$ for $5 \mathrm{~min}$. The PCR products were run on a $1.5 \%$ agarose gel, and the band intensity was measured and normalized to the internal control $\beta$-actin.

Western blotting. Total protein was extracted from HEp2 cells. In brief, cells were lysed using $250 \mu \mathrm{l}$ of RIPA lysis buffer and then underwent a process of homogenization for $10 \mathrm{~min}$, ice-bath for $1 \mathrm{~h}$ and centrifugation at 12,000 x g for $30 \mathrm{~min}$ at $4^{\circ} \mathrm{C}$. The supernatant was finally collected, and the protein concentration was determined by the BCA protein assay system (Pierce Biotechnology, Inc., Rockford, IL, USA). Extracts equivalent to $50 \mu \mathrm{g}$ of total protein were separated by sodium dodecyl sulfate (SDS)-polyacrylamide gel electrophoresis and transferred onto nitrocellulose membranes. After being blocked overnight at $4^{\circ} \mathrm{C}$ with $1 \mathrm{X}$ PBS, $0.1 \%$ Tween-20 and 5\% non-fat milk, the membranes were incubated with the primary anti- 


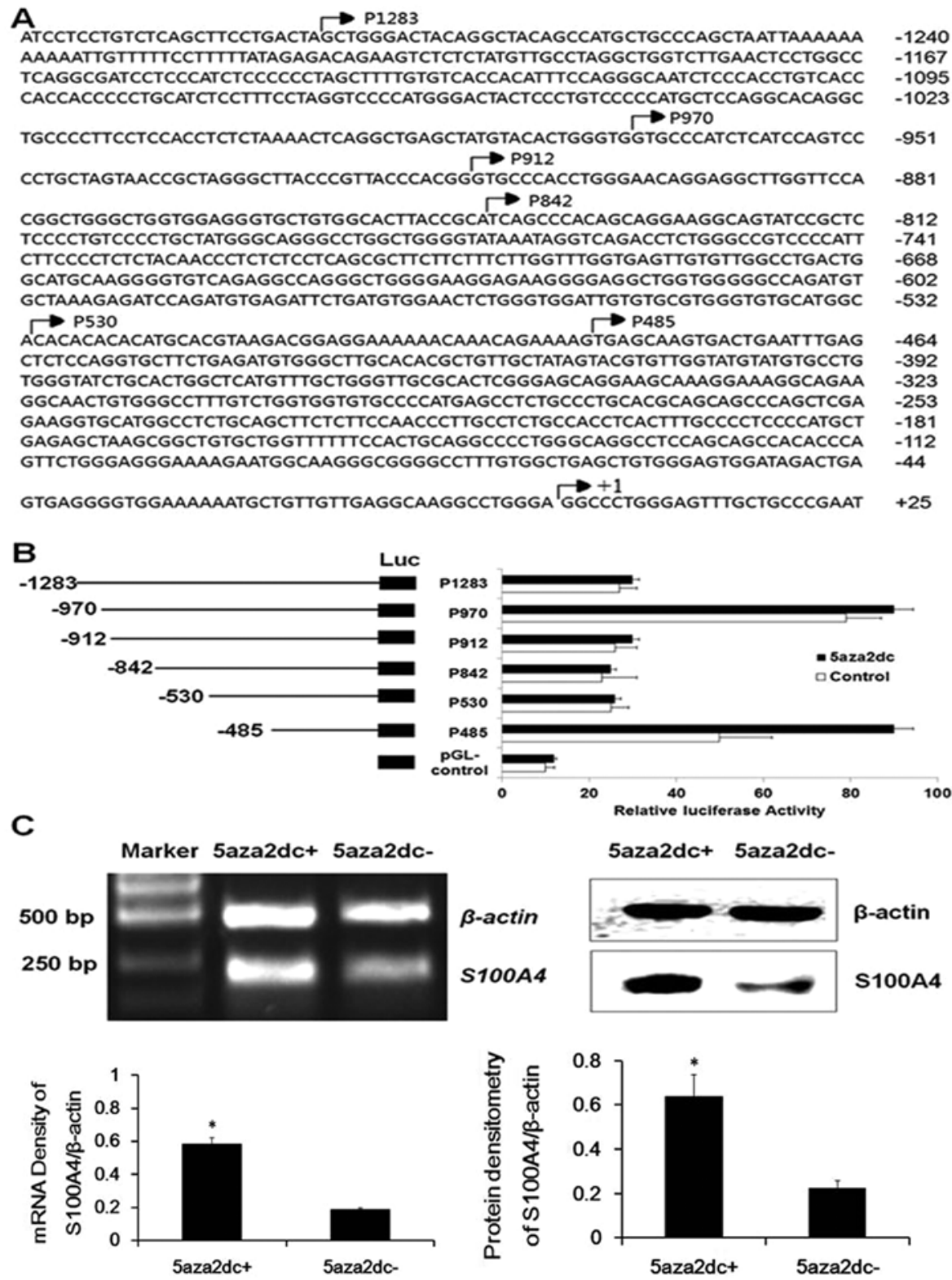

Figure 1. Analysis of the promoter activities and expression of the human S100A4 gene. (A) Illustration of over $1.2 \mathrm{~kb}$ fragment of the S100A4 5'-flanking sequence. (B) The region $-485-+73$ is responsible for the upregulation of the human S100A4 gene in the 5-Aza-induced HEp2 cells. The pGL3-Basic-S100A4 5'-UTR vectors were transferred into HEp2 cells, respectively. After $72 \mathrm{~h}$ of transient transfection into HEp2 cells, the activities of luciferase reporter genes were detected. All values are the average of 3 independent experiments (means \pm standard deviation), and data were analyzed using SPSS 13.0 statistical software. The pGL3-Basic vector was used as control. (C) 5-Aza induces the overexpression of S100A4 in HEp2 cells. RT-PCR and western blot methods were used to determine the mRNA and protein levers of S100A4, respectively. $\beta$-actin was used for normalization. Prior to detection, the human HEp2 cells were treated with 5-Aza for over 3 days. The data were obtained from 3 experiments. 5-Aza, 5aza2dc.

bodies against S100A4 $(1: 2,000)$ or $\beta$-actin $(1: 1,000)$ for $3 \mathrm{~h}$ at room temperature, washed twice and then incubated with the secondary horseradish peroxidase-conjugated goat anti-rabbit antibody (1:5,000; Zhongshan, China) for $2 \mathrm{~h}$ at room temperature. Immunodetection was performed with chemiluminescence (ECL reagent; Beyotime, China), and the membranes were exposed to film. The densities of the bands on the filters were quantified by densitometric analysis using the UVP GelWorks ID advanced version 2.5 software (Bio-Rad, USA).

Bisulfite modification and bisulfite-specific PCR. Genomic DNA isolated from the HEp2 cells was used to detect the methylation status of the $S 100 A 4$ promoter. In brief, $\sim 1 \mu \mathrm{g}$ of genomic DNA was bisulfite-modified using the EZ DNA Methylation-Gold ${ }^{\mathrm{TM}}$ kit (Zymo Research, Orange, CA, USA) according to the manufacturer's recommendation. Based on the functional promoter sequence of the S100A4 gene, the primers (forward, 5'-TAGAAAAGTGAGTAAGTGATTGA-3' and reverse, 5'-TC TACCTTTCCTTTACTTCCTAC-3') were used in bisulfite-specific PCR detection, and the amplified fragment was $170 \mathrm{bp}$. The PCR reaction was performed in a $25-\mu 1$ reaction system, starting with denaturation at $94^{\circ} \mathrm{C}$ for $4 \mathrm{~min}$, then 42 cycles of denaturation at $94^{\circ} \mathrm{C}$ for $30 \mathrm{sec}$, annealing at $53^{\circ} \mathrm{C}$ for $30 \mathrm{sec}$, extension at $72^{\circ} \mathrm{C}$ for $45 \mathrm{sec}$, followed by an extra extension at $72^{\circ} \mathrm{C}$ for $5 \mathrm{~min}$. The BSP products were then cloned into a T-vector (Takara, Japan), and 


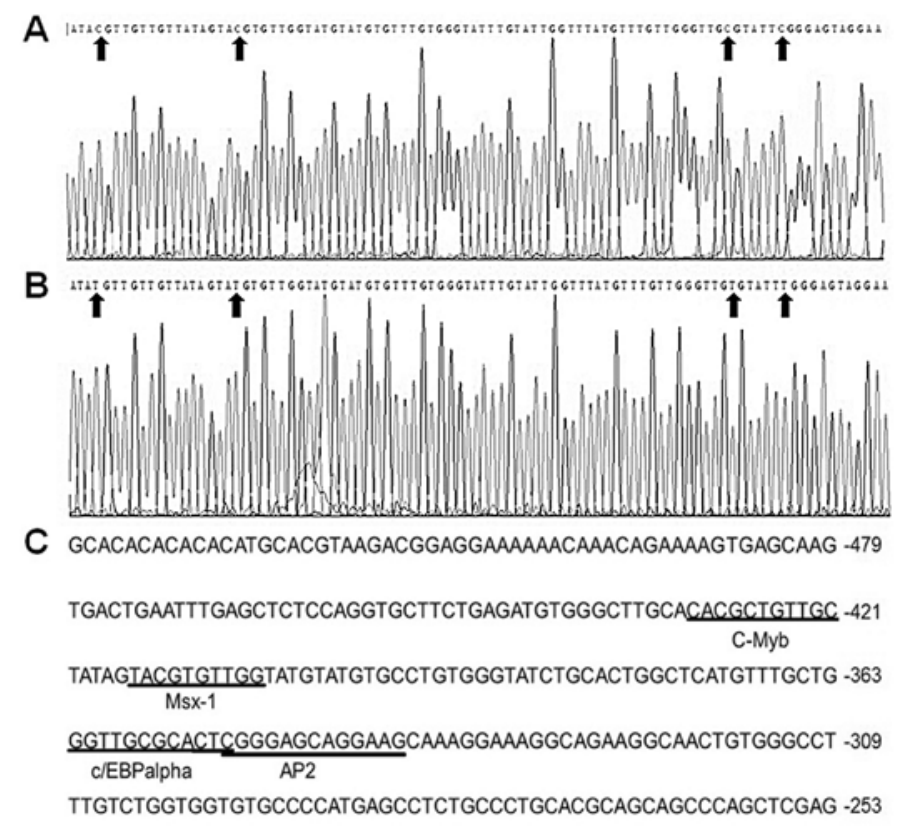

AAGGTGCATGGCCTCTGCAGCTTCTCTTCCAACCCTTGCCTCTGCCACCTCACT -199

Figure 2.5-Aza alters the methylation status of the cytosines in the region $-485-+73$ which harbors several potential cis-acting elements in HEp2 cells. (A) The cytosines at $\mathrm{CpG}$ were methylated in the region $-485-+73$ in the HEp2 cells prior to 5-Aza treatment. (B) The methylation-free cytosines at CpG in the region $-485-+73$ in the HEp2 cells were restored by 5 -Aza. BSP-based sequencing was used to detect the methylation status of the 4 CpG sites in the region $-485-+73$ in the HEp2 cells prior to or after 5-Aza treatment. The arrows indicate the methylation status at the CpG sites. (C) The 4 CpGs are located in the sequences of 4 potential transcription factor binding sites predicted in the region $-485-+73$. c-Myb, C/EBP $\alpha, \mathrm{Ap} 2$ and Msx-1 potential transcription factor binding sites were predicted using P-Match ${ }^{\mathrm{TM}}$ software.

JM109 E. coli competent cells (Takara, Japan) were used for transformation according to the manufacturer's instructions.

Bioinformatics. The human S100A4 promoter sequence was obtained from e-Ensembl with the accession no. ENST00000368715. The binding sites were predicted by using the online P-Match ${ }^{\mathrm{TM}}$ program (http://www.gene-regulation. com/pub/programs.html\#pmatch).

Electromobility shift assay (EMSA). Non-denatured cellular nuclear proteins were prepared using a nuclear protein extraction kit according to the manufacturer's instructions (Active Motif, Carlsbad, CA, USA). EMSA was carried out using a commercially available LightShift Chemiluminescent EMSA kit (Pierce Biotechnology, Inc.). The probes used in EMSA were synthesized by Sangene (Beijing, China), and the sequences were as follows: c-Myb wild-type, 5'-tgcacacgctgttgctatagta-3' and mutant, 5'-tgcacacgctgaatctatagta-3'; cEBp $\alpha$ wild-type, 5'-gggttgcgcactcgggagcagg-3' and mutant, 5'-gggaatcgcactcgggagcagg-3'. Two micrograms of nuclear protein extracts was incubated with 3'-end-biotin-labeled S100A4 DNA probes in binding buffer for $30 \mathrm{~min}$ on ice, separated on a $6 \%$ non-denaturing polyacrylamide gel in $0.5 \mathrm{X} \mathrm{TBE}$, and then transferred onto a nylon membrane and fixed by ultraviolet cross-linking. The biotin-labeled probes were detected with streptavidin-horseradish peroxidase (Pierce Biotechnology, Inc.). For the competition experiments, a 100-fold excess of unlabeled doubled-stranded cold probes was added to the binding reaction. To determine the effect of the antibodies on protein-DNA binding, $1 \mu \mathrm{g}$ primary antibodies against c-Myb, $\mathrm{C} / \mathrm{EBP} \alpha, \mathrm{Ap} 2$ and Msx-1 (Santa Cruz Biotechnology, Inc.) was incubated with the nuclear extracts for $30 \mathrm{~min}$ on ice prior to the addition of the biotin-labeled DNA probes, respectively.

Chromatin immunoprecipitation (ChIP) assay. The ChIP-IT kit was purchased from Active Motif, and the ChIP assay was carried out according to the manufacturer's protocol. One microgram of antibodies against c-Myb or C/EBP $\alpha$ was utilized for immunoprecipitation. After proteinase $\mathrm{K}$ digestion and DNA precipitation, $3 \mu \mathrm{g} / \mathrm{l}$ of diluted DNA was used for PCR. The primer sequences for amplifying the $S 100 A 4$ promoter and GAPDH were as follows: S100A4 forward, 5'-CAAACAG AAAAGTGAGCAAGTGAC-3' and reverse, 5'-AGAGGCAA GGGTTGGAAGAG-3' to generate a 289-bp fragment; GAPDH forward, 5'-CGACCACTTTGTCAAGCTCA-3' and reverse, 5'-AGGGGTCTACATGGCAACTG-3' to generate a 332-bp fragment.

Statistical analysis. Unless otherwise stated, each experiment was performed for a minimum of 3 times. Data were subjected to statistical analysis using SPSS 13.0 software and are shown as means \pm standard error of the mean (SEM). Differences in mean values were analyzed using one-way analysis of variance (ANOVA) or t-test as appropriate. Statistical significance was assumed for a two-tailed $\mathrm{P}<0.05$.

\section{Results}

S100A4 promoter region $-485-+73$ harbors major transcription factor binding sites related to the methylation status in laryngeal cancer cells. In search of the potential cis-acting elements of the S100A4 promoter, several constructs including 


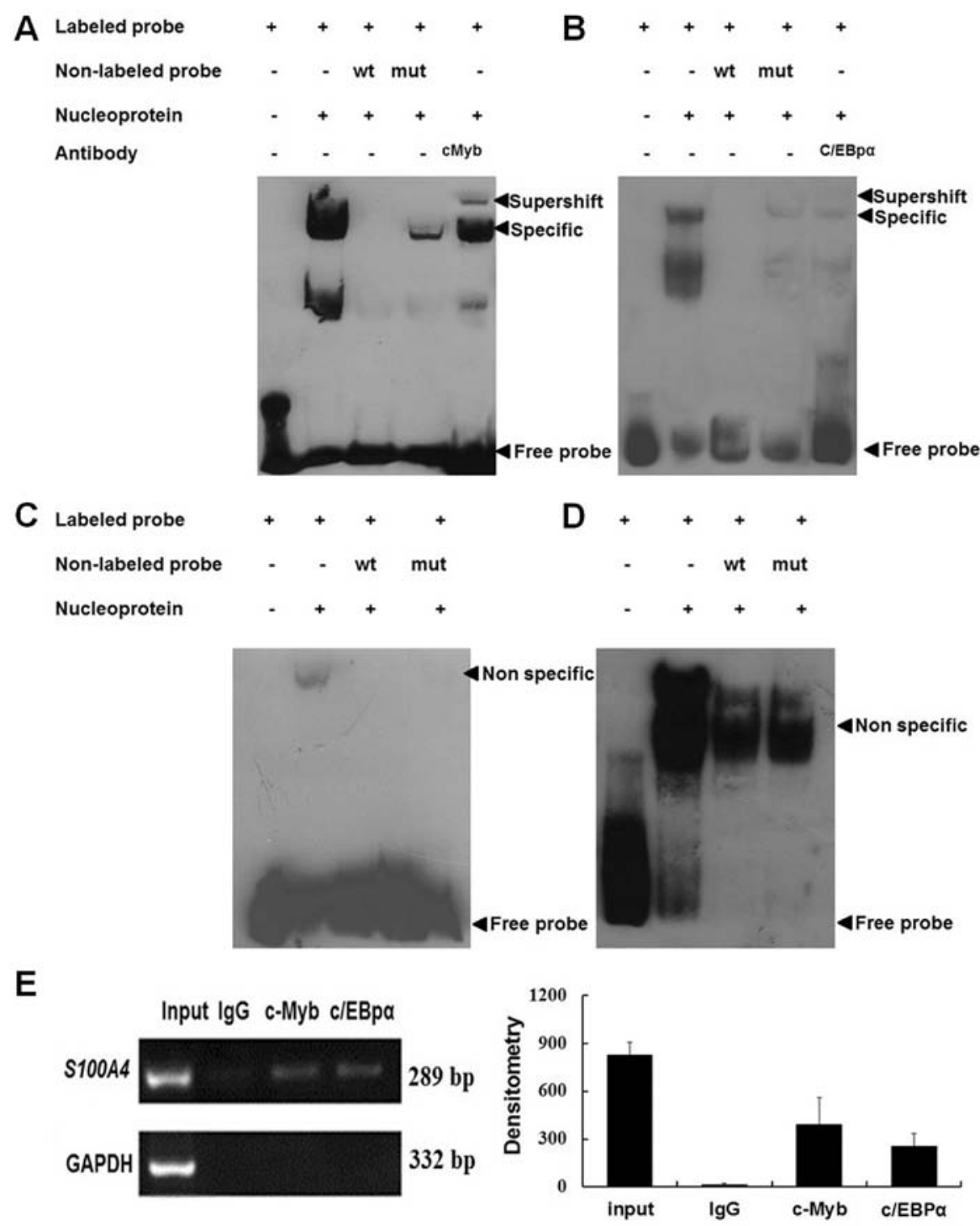

Figure 3. c-Myb and C/EBP $\alpha$ are novel transcription factors of S100A4. (A-D) c-Myb and C/EBP $\alpha$, but not Ap2 and Msx-1, bind the region $-485-+73$ of S100A4 in vitro. The EMSA results indicated that (A) c-Myb and (B) C/EBP $\alpha$ could bind S100A4 in vitro but not (C) Ap2 and (D) Msx-1. (E) c-Myb and C/EBP $\alpha$ bind the region $-485-+73$ of S100A4 in vitro as well. The binding of c-Myb and C/EBP $\alpha$ to the region $-485-+73$ of S100A4 in vitro was confirmed by ChIP. Input serves as the positive control. IgG and GAPDH were used as negative and internal controls in the immunoprecipitation reaction and PCR, respectively. EMSA, electromobility shift assay; ChIP, chromatin immunoprecipitation.

the region $-485-+73$ of $\$ 100 A 4$ were generated (Fig. 1A), and transient transfection was then performed to assess their relative reporter gene activities. As shown in Fig. 1B, under basal conditions, the constructs p970-luc and p485-luc in HEp2 cells produced much higher luciferase activities than those of the other truncated vectors $(\mathrm{P}<0.05)$, indicating that the region $-485-+73$ harbors the positive cis-acting elements, and the fragment $-486--530$ the negative cis-acting elements. Following a 72-h exposure to $4 \mu \mathrm{M} 5$-Aza, the HEp2 cells transfected by p485-luc resulting in a significantly high level of luciferase activity when compared to the other groups $(\mathrm{P}<0.05)$. RT-PCR and western blot results also indicated that 5-Aza induced significant overexpression of S100A4 both at the mRNA and protein levels (Fig. $1 \mathrm{C}, \mathrm{P}<0.05$ ). The MSP-based sequencing result showed that the cytosines of the dinucleotide CG at position $-485-+73$ were methylated in the untreated HEp2 cells and the methyl groups of the cytosines were removed in HEp2 cells treated with 5-Aza (Fig. 2A and B). These findings suggest that demethylation increases the expression of endogenous transcription factor binding to the area and results in the methylation-free status in the region of S100A4 as well. Therefore, there could exist important cisacting elements within the promoter region $-485-+73$, which is affected by the methylation status of either the gene itself or the upstream genes regulating the transcription of S100A4.

Putative cis-acting elements in the S100A4 promoter region $-485-+73$ are predicted. The P-Match software was used to predict the cis-acting elements in the $5100 A 4$ promoter region $-485-+73$. The result indicted that this region contained the binding motifs of certain transcription factors including c-Myb, C/EBP $\alpha$, Ap2 and Msx-1 (Fig. 2C). The cytosines in these binding sites were methylated in HEp2 cells (Fig. 2A and B). 
A
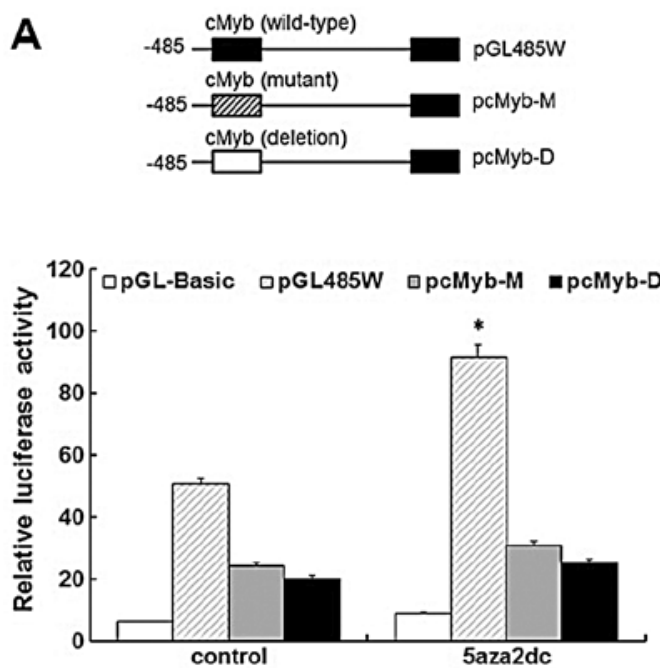

B
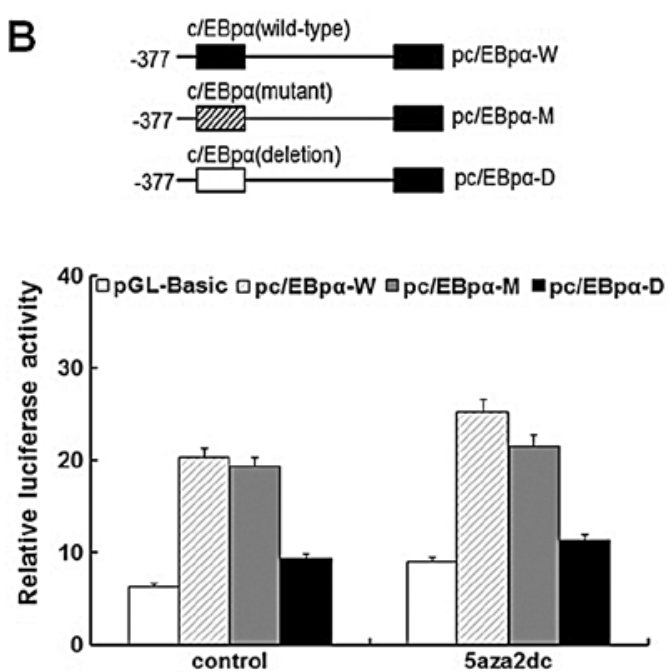

Figure 4. c-Myb is suggested to be a key transcription factor in region $-485-+73$ of S100A4 in methylation-free HEp2 cells. (A) The mutant vector (pcMyb-M) interfered with its binding to S100A4. (B) The mutant vector ( $\mathrm{pC} / \mathrm{EBP} \alpha-\mathrm{M})$ did not affect its binding to S100A4. The c-Myb and C/EBP $\alpha$ wild-type, mutant and deletion luciferase reporter vectors were constructed. After transfection, the luciferase activity of the different reporter genes in HEp2 cells prior to or after 5-Aza treatment was detected. All values are the average of 3 independent experiments (means \pm standard deviation), and data were analyzed using SPSS 13.0 statistical software. 5-Aza, 5aa2dc.

$c-M y b$ and $C / E B P \alpha$ bind to the cis-acting elements in the S100A4 promoter region $-485-+73$. To confirm the binding of the $5100 A 4$ gene to c-Myb, C/EBP $\alpha$, Ap2 and Msx-1, EMSA and ChIP methods were used. As shown in Fig. 3A-D, the binding of c-Myb and $\mathrm{C} / \mathrm{EBP} \alpha$, but not Ap2 and Msx-1, to $S 100 A 4$ was detected when the wild-type probes were used. The addition of the cold self-competitors weakened the binding, and the mutant competitor could not abolish the binding. Supershift bands were also observed when c-Myb and $\mathrm{C} / \mathrm{EBP} \alpha$ antibodies were applied. These results indicated that c-Myb and C/EBP $\alpha$ were able to bind the S100A4 promoter region $-485-+73$ in vitro. ChIP results showed that both $\mathrm{c}-\mathrm{Myb}$ and $\mathrm{C} / \mathrm{EBP} \alpha$ antibodies, but not non-immune $\mathrm{IgG}$, could immunoprecipitate c-Myb and $\mathrm{C} / \mathrm{EBP} \alpha$ proteins binding to the same region in vitro (Fig. 3E).

c-Myb is a major trans-acting element of S100A4 in 5-Aza-induced HEp2 cells. To simulate the effects of demethylation on methylated sites within the c-Myb and $\mathrm{C} / \mathrm{EBP} \alpha$ response elements of $S 100 A 4$, we constructed the mutant and deletion reporter vectors of c-Myb and $\mathrm{C} / \mathrm{EBP} \alpha$ motifs corresponding to the methylated sites, named pcMyb-M, pcMyb-D, $\mathrm{pC} / \mathrm{EBP} \alpha-\mathrm{M}$ and $\mathrm{pC} / \mathrm{EBP} \alpha-\mathrm{D}$, respectively. These vectors were then transiently transfected into HEp2 cells to evaluate their relative reporter gene activities. The mutant and deletion fragments (pcMyb-M/pcMyb-D) showed a significant decrease in the reporter activities compared to the wild-type construct in the 5-Aza-treated and -untreated groups $(\mathrm{P}<0.05)$, confirming that the mutation and deletion constructs interfere with the binding of c-Myb to S100A4 (Fig. 4A). Fig. 4A also showed the significant difference in the luciferase activities of the reported genes between 5-Aza-treated and -untreated HEp2 cells transfected by the wild-type construct, which implies that the overexpression of the endogenous c-Myb gene in HEp2 cells induced by 5-Aza may lead to the overexpression of the reporter genes in the 5-Aza-treated group. As shown in Fig. 4B, no significant luciferase activity of the reporter genes on $\mathrm{C} / \mathrm{EBP} \alpha$ wild-type vectors was observed, which indicates that 5-Aza does not induce the overexpression of endogenic $\mathrm{C} / \mathrm{EBP} \alpha$ in HEp2 cells, even though the $\mathrm{C} / \mathrm{EBP} \alpha$-binding sites in the $S 100 A 4$ promoter region $-485-+73$ were demethylated in HEp2 cells. There was also no significant difference in luciferase activity of the reporter genes on $\mathrm{C} / \mathrm{EBP} \alpha$ wild-type and mutant-type vectors between the 5-Aza-treated or -untreated groups, which indicates that the mutant sites reflecting the methylated ones in $\mathrm{C} / \mathrm{EBP} \alpha$ do not affect the binding of $\mathrm{C} / \mathrm{EBP} \alpha$ to S100A4 in the HEp2 cells. Yet, the $\mathrm{C} / \mathrm{EBP} \alpha$ deletion vector showed a significant decrease in the reporter activity when compared to the wild-type and mutant-type vectors in the 5-Aza-treated and -untreated groups $(\mathrm{P}<0.05)$, which suggests that the $\mathrm{C} / \mathrm{EBP} \alpha$ response element but not its cytosine methylation status plays an important role in the transcriptional regulation of the S100A4 gene.

\section{Discussion}

S100A4 is overexpressed in many cancer types including laryngeal carcinoma, which indicates that this gene plays an important role in carcinogenesis. As demonstrated in our previous study, there is a strong correlation between overexpression and hypomethylation of S100A4 in laryngeal cancer tissues (15). In addition, similar phenomena are found in endometrial, pancreatic ductal, colon and ovarian carcinomas (17-19). At present, little is known concerning the molecular mechanisms behind the transcriptional regulation of S100A4 expression-related hypomethylation. In ovarian cancer, the upregulation of S100A4 expression is associated with hypomethylation of CpG sites in the first intron of S100A4, and hypoxia can reduce the methylation of hypoxia-response elements (HRE) of the S100A4 gene in a time-dependent fashion, in association with the increased binding of HIF-1 $\alpha$ to a methylation-free HRE (20). Therefore, we speculate that the methylation-free sites in the $S 100 A 4$ promoter region can 
increase the binding of the corresponding transcription factors to S100A4 in laryngeal cancer.

In the present study, we cloned and characterized over $1.2 \mathrm{~kb}(-1283$ to +73$)$ region lying upstream of the human S100A4 transcription start site to determine the major cisacting elements, particularly those related to the methylation status in HEp2 cells. As a result, we revealed the existence of potential positive and negative $c i s$-acting elements in the corresponding regions of the $S 100 A 4$ promoter. Further investigation was focused on the region $-485-+73$ as the methylation status of the region significantly affected the expression of S100A4. Notably, the cytosines in the region were methylated in the HEp2 cells, and 5-Aza altered the methylation status (Fig. 2A and $\mathrm{B})$. In this region, there were several putative cis-acting elements such as c-Myb, C/EBP $\alpha$, Ap2 and Msx-1. EMSA and ChIP results confirmed the binding of c-Myb and C/EBP $\alpha$ to S100A4. Comparing the luciferase activity of the reporter genes on the mutant, deletion and wild-type vectors in the HEp2 cells treated by 5 -Aza or not, we found that only c-Myb promoted S100A4 expression in the methylation-free HEp2 cells. We thus speculated that the overexpression of endogenous c-Myb in HEp2 cells induced by 5-Aza plays a partial role in the S100A4 expression in the methylation-free HEp2 cells. Nevertheless, the c-Myb response element in the region $-485-+73$ is the major element in the regulation of S100A4 expression in HEp2 cells when the methylation status is changed. The c-Myb proto-oncogene product $(c-M y b)$ plays an important role in the proliferation of immature hematopoietic, colonic epithelial, and mammary epithelial cells and also in the differentiation of hematopoietic cells (21-24). The identified target genes of $c-M y b$ include c-myc, Bcl-2 and Gbx2, which are involved in cell cycle control, blockage of apoptosis, and growth and differentiation control, respectively (25-28). Presently, no related study on the association of $c-M y b$ with the development of laryngeal cancer has been reported. Similar to our study, Wang et al found that methylation of the $\mathrm{CpG}$ site in the WNT5A promoter influences its binding to c-Myb and 5-Aza increased the expression of WNT5A, which suggests that binding of c-Myb to WNT5A is methylation-sensitive and is inhibited by the methylation of the CpG site in prostate cancer cells (29). Campanero et al found that methylation of the E2F elements in the c-Myc and c-Myb promoters significantly inhibited the binding of E2F1, and suggested that methylation of the c-Myb element from the S100A4 promoter may affect its binding to c-Myb (30). In the present study, S100A4 was confirmed as a novel target of $\mathrm{c}-\mathrm{Myb}$ and $\mathrm{C} / \mathrm{EBP} \alpha$, which enriched the regulatory network in which c-Myb and C/EBP $\alpha$ were involved. Fang et al found that 663 transcripts including c-Myb and S100A4 were regulated by CypB knockdown, and that many of these gene products contributed to cell proliferation, cell motility and tumorigenesis, which indicates that the co-overexpression of $c-M y b$ and S100A4 participates in the pathogenesis of breast cancer (31). Whether CypB directly regulates c-Myb and S100A4, respectively, or regulates S100A4 via c-Myb is not known.

In conclusion, in the present study, we first revealed that c-Myb and C/EBP $\alpha$ can target the S100A4 gene through binding the corresponding cis-acting motifs in the sequence $-485-+73$. The overexpression of the S100A4 gene is caused by demethylation of the c-Myb motif and possible overexpression of the $c-M y b$ gene in methylation-free HEp2 cells. Whether the overexpression of the $c-M y b$ gene in the 5-Aza-induced HEp2 cells was caused by the demethylation of $c-M y b$ gene itself or of other upstream genes regulating $c-M y b$ or both warrants further investigation. The identification and functional study of potential negative response elements in the S100A4 promoter region $-486--530$ will be the focus of subsequent research by our group.

\section{Acknowledgements}

This study was supported by the National Natural Science Foundation of China (81172577 and 81301766), and the Natural Science Foundation of Liaoning Province (20092110). J.L. obtained the grant (81301766), which supports the study of S100A4 on the invasiveness of laryngeal cancer and was approved on August, 2013.

\section{References}

1. Ragin CC, Modugno F and Gollin SM: The epidemiology and risk factors of head and neck cancer: a focus on human papillomavirus. J Dent Res 86: 104-114, 2007.

2. Li DW, Gao S, Shen B and Dong P: Effect of apoptotic and proliferative indices, P-glycoprotein and survivin expression on prognosis in laryngeal squamous cell carcinoma. Med Oncol 28 (Suppl 1): S333-S340, 2011.

3. Holgersson G, Ekman S, Reizenstein J, et al: Molecular profiling using tissue microarrays as a tool to identify predictive biomarkers in laryngeal cancer treated with radiotherapy. Cancer Genomics Proteomics 7: 1-7, 2010.

4. Zou J, Yang H, Chen F, et al: Prognostic significance of fascin-1 and E-cadherin expression in laryngeal squamous cell carcinoma. Eur J Cancer Prev 19: 11-17, 2010.

5. Waha A, Felsberg J, Hartmann W, et al: Frequent epigenetic inactivation of the chaperone $S G N E 1 / 7 B 2$ in human gliomas. Int J Cancer 131: 612-622, 2012.

6. Robertson KD: DNA methylation and human disease. Nat Rev Genet 6: 597-610, 2005.

7. Wilson IM, Davies JJ, Weber M, et al: Epigenomics: mapping the methylome. Cell Cycle 5: 155-158, 2006.

8. Malone CS, Miner MD, Doerr JR, et al: $\mathrm{C}^{\mathrm{m}} \mathrm{C}(\mathrm{A} / \mathrm{T}) \mathrm{GG}$ DNA methylation in mature $\mathrm{B}$ cell lymphoma gene silencing. Proc Natl Acad Sci USA 98: 10404-10409, 2001.

9. Esteller M: Cancer epigenetics: DNA methylation and chromatin alterations in human cancer. Adv Exp Med Biol 532: 39-49, 2003.

10. Waraya M, Yamashita K, Katoh H, et al: Cancer specific promoter CpG Islands hypermethylation of HOP homeobox (HOPX) gene and its potential tumor suppressive role in pancreatic carcinogenesis. BMC Cancer 12: 397, 2012.

11. Abildgaard MO, Borre M, Mortensen MM, et al: Downregulation of zinc finger protein 132 in prostate cancer is associated with aberrant promoter hypermethylation and poor prognosis. Int $\mathbf{J}$ Cancer 130: 885-895, 2012.

12. Lleras RA, Adrien LR, Smith RV, et al: Hypermethylation of a cluster of Krüppel-type zinc finger protein genes on chromosome 19q13 in oropharyngeal squamous cell carcinoma. Am J Pathol 178: 1965-1974, 2011.

13. Rosty C, Ueki T, Argani P, et al: Overexpression of S100A4 in pancreatic ductal adenocarcinomas is associated with poor differentiation and DNA hypomethylation. Am J Pathol 160: 45-50, 2002.

14. Choi JY, James SR, Link PA, et al: Association between global DNA hypomethylation in leukocytes and risk of breast cancer. Carcinogenesis 30: 1889-1897, 2009.

15. Liu J, Guo Y, Fu S, Yang M, Sun KL and Fu WN: Hypomethylationinduced expression of S100A4 increases the invasiveness of laryngeal squamous cell carcinoma. Oncol Rep 23: 1101-1107, 2010.

16. Burke E and Barik S: Megaprimer PCR: application in mutagenesis and gene fusion. Methods Mol Biol 226: 525-532, 2003.

17. Xie R, Loose DS, Shipley GL, Xie S, Bassett RL Jr and Broaddus RR: Hypomethylation-induced expression of S100A4 in endometrial carcinoma. Mod Pathol 20: 1045-1054, 2007. 
18. Sato N, Maitra A, Fukushima N, et al: Frequent hypomethylation of multiple genes overexpressed in pancreatic ductal adenocarcinoma. Cancer Res 63: 4158-4166, 2003.

19. Nakamura $\mathrm{N}$ and Takenaga K: Hypomethylation of the metastasis-associated S100A4 gene correlates with gene activation in human colon adenocarcinoma cell lines. Clin Exp Metastasis 16: 471-479, 1998.

20. Horiuchi A, Hayashi T, Kikuchi N, et al: Hypoxia upregulates ovarian cancer invasiveness via the binding of HIF-1 $\alpha$ to a hypoxia-induced, methylation-free hypoxia response element of S100A4 gene. Int J Cancer 131: 1755-1767, 2012.

21. Mucenski ML, McLain K, Kier AB, et al: A functional c-myb gene is required for normal murine fetal hepatic hematopoiesis. Cell 65: 677-689, 1991.

22. Malaterre J, Carpinelli M, Ernst M, et al: c-Myb is required for progenitor cell homeostasis in colonic crypts. Proc Natl Acad Sci USA 104: 3829-3834, 2007.

23. Drabsch Y, Hugo H, Zhang R, et al: Mechanism of and requirement for estrogen-regulated $M Y B$ expression in estrogenreceptor-positive breast cancer cells. Proc Natl Acad Sci USA 104: 13762-13767, 2007.

24. Bender TP, Kremer CS, Kraus M, Buch T and Rajewsky K: Critical functions for c-Myb at three checkpoints during thymocyte development. Nat Immunol 5: 721-729, 2004.
25. Nakagoshi H, Kanei-Ishii C, Sawazaki T, Mizuguchi G and Ishii S: Transcriptional activation of the c-myc gene by the c-myb and B-myb gene products. Oncogene 7: 1233-1240, 1992.

26. Frampton J, Ramqvist T and Graf T: v-Myb of E26 leukemia virus up-regulates bcl-2 and suppresses apoptosis in myeloid cells. Genes Dev 10: 2720-2731, 1996.

27. Taylor D, Badiani P and Weston K: A dominant interfering Myb mutant causes apoptosis in T cells. Genes Dev 10: 2732-2744, 1996.

28. Kowenz-Leutz E, Herr P, Niss K and Leutz A: The homeobox gene $G B X 2$, a target of the myb oncogene, mediates autocrine growth and monocyte differentiation. Cell 91: 185-195, 1997.

29. Wang Q, Williamson M, Bott S, et al: Hypomethylation of WNT5A, CRIP1 and S100P in prostate cancer. Oncogene 26: 6560-6565, 2007.

30. Campanero MR, Armstrong MI and Flemington EK: CpG methylation as a mechanism for the regulation of E2F activity. Proc Natl Acad Sci USA 97: 6481-6486, 2000.

31. Fang F, Flegler AJ, Du P, Lin S and Clevenger CV: Expression of cyclophilin $\mathrm{B}$ is associated with malignant progression and regulation of genes implicated in the pathogenesis of breast cancer. Am J Pathol 174: 297-308, 2009. 